\title{
Common Fixed Point Theorems for Weakly Subsequentially Continuous Mappings in Modified Intuitionistic Fuzzy Metric Spaces
}

\author{
Naeem Salem ${ }^{1, *}$, Said Beloul ${ }^{2}$ \\ ${ }^{1}$ Department of Mathematics, University of Management and Technology, Lahore, Pakistan \\ ${ }^{2}$ Department of Mathematics, Faculty of Exact Sciences, El-Oued University, P.O.Box789, El-Oued 39000, Algeria
}

Copyright (c) 2017 by authors, all rights reserved. Authors agree that this article remains permanently open access under the terms of the Creative Commons Attribution License 4.0 International License

\begin{abstract}
The aim of this paper is to establish some common fixed point results for two weakly subsequentially continuous and compatible of type (E) pairs of self mappings via implicit relation in modified intuitionistic fuzzy metric spaces, also we give an example to illustrate our results.
\end{abstract}

Keywords Common Fixed Point, Weakly Subsequentially Continuous, Compatible of Type (E), Modified IFMS

\section{Introduction}

The notion of intuitionistic fuzzy sets was been introduced by Atanassov [1], it can be considered as a generalization to fuzzy sets concept due to Zadeh [30]. Later Çoker [6] introduced a topology on intuitionistic fuzzy sets. Park [21] introduced the notion of intuitionistic fuzzy metric spaces as a generalization to fuzzy metric spaces, which is a combination between intuitionistic fuzzy sets and the concept of a fuzzy metric space given by George and Veeramani [9], many authors established some results concerning fixed point in such spaces, see for example [2, 5, 12, 14, 19, 29].

Meanwhile, jungck[16] defined the concept of compatible mappings, Jungck and Roadhes [17] generalized the last concept to the weakly compatible mappings, which is weaken than the compatible ones. Mishra et al [20] generalized the concept of compatibility in the setting of fuzzy metric spaces, he obtained some common fixed point theorems for compatible mappings in such spaces. Recently Bouhadjera and Godet Tobie [4] introduced the concept of subsequential continuity and utilized it with the concept of subcompatible mappings to establish a common fixed point, later Imdad et al.[13] improved these results and replaced subcompatibility by compatibility and subsequential continuity by reciprocal continuity, more recently, Gopal and Imdad [11] combined subsequential continuous maps with compatible maps concept to obtain some results in fuzzy metric spaces. In present work, we will generalize certain definitions to intuitionistic fuzzy metric spaces in order to obtain some common fixed point theorems by combining the concept of weakly subsequentially continuous mappings due to second author [3] with compatible of type (E) mappings given by Singh et al.[27, 28].

\section{Preliminaries}

Lemma 2.1. [7] Let $t L^{*}$ be a non empty set such:

$$
L^{*}=\left\{\left(x_{1}, x_{2}\right) \in[0,1]^{2}, \quad x_{1}+x_{2} \leq 1\right\},
$$

also define an operation $\leq_{L^{*}}$ as follows:

$$
\left(x_{1}, x_{2}\right) \leq_{L^{*}}\left(y_{1}, y_{2}\right) \Longleftrightarrow\left(x_{1} \leq x_{2}\right) \text { and }\left(y_{1} \geq y_{2}\right),
$$


for all $\left(x_{1}, x_{2}\right),\left(y_{1}, y_{2}\right) \in[0,1]^{2}$. So $\left(L^{*}, \leq_{L^{*}}\right)$ is a complete lattice.

Definition 2.1. [1] We called $n$ intuitionistic fuzzy set $A_{\zeta, \eta}$ in universe $U$ every objet $A_{\zeta, \eta}=\left\{\left(\zeta_{A}(u), \eta_{A}(u)\right), u \in U\right\}$, where $u \in U$, for all $u \in U, \zeta_{A}(u) \in[0,1]$ and $\eta_{A}(u) \in[0,1]$ are said to be the membership degree and the non-membership degree, respectively, of $u \in A_{\zeta, \eta}$ and furthermore they satisfy $\zeta_{A}(u)+\eta_{A}(u) \leq$ 1.

For every $z_{i}=\left(x_{i}, y_{i}\right) \in L^{*}$, if $c_{i} \in[0,1]$ such that $\sum_{j=1}^{n} c_{j}=1$, then we have:

$$
z_{1}\left(x_{1}, y_{1}\right)+\ldots+z_{n}\left(x_{n}, y_{n}\right)=\sum_{j=1}^{n} z_{j}\left(x_{j}, y_{j}\right)=\left(\sum_{j=1}^{n} c_{j} x_{j}, \sum_{j=1}^{n} c_{j} y_{j}\right) \in L^{*}
$$

Its units are given by $0_{L^{*}}=(0,1)$ and $1_{L^{*}}=(1,0)$. A triangular norm $T=*$ on $[0,1]$ is considered as an commutative, associative and increasing mapping $T:[0,1]^{2} \rightarrow[0,1]$ such that $T(1, x)=1 * x=x$, for all $x \in[0,1]$. The same, a triangular co-norm $S=\diamond$ is a commutative, associative and increasing mapping $S:[0,1]^{2} \rightarrow[0,1]$ such that $S(0, x)=0 \diamond x=x$, for all $x \in[0,1]$.

Definition 2.2. [7] 8] Let $\mathcal{T}$ be a continuous $t$-norm on $L^{*}$, then $\mathcal{T}$ is a continuous t-representable if and only if there exist a continuous $t$-norm $*$ and a continuous $t$-conorm $\diamond$ on $[0,1]$ satisfy:

$$
\mathcal{T}(x, y)=\left(x_{1} * y_{1}, x_{2} \diamond y_{2}\right)
$$

for all $\left.x=\left(x_{1}, x_{2}\right), y=y_{1}, y_{2}\right) \in L^{*}$.

Now, let $\left\{\mathcal{T}_{n}\right\}$ be a sequence such that $\left\{\mathcal{T}_{1}=\mathcal{T}\right\}$ and for $n>1$ and $x(i) \in L^{*}$,

$$
\mathcal{T}^{n}\left(x^{(1)}, \ldots, x^{(n+1)}=\mathcal{T}\left(\mathcal{T}^{n-1}\left(x^{(1)}, \ldots, x^{(n)}\right), x^{(n+1)}\right) .\right.
$$

Definition 2.3. [8] A mapping $\mathcal{T}:\left(L^{*}\right)^{2} \rightarrow L^{*}$ is called a triangular norm on $L^{*}$ if for all $(x, y, z) \in\left(L^{*}\right)^{3}$, the following conditions hold:

1. $\mathcal{T}\left(x, 1_{L^{*}}\right)=x$.

2. $\mathcal{T}(x, y)=\mathcal{T}(y, x)$.

3. $\mathcal{T}(x, \mathcal{T}(y, z))=\mathcal{T}((\mathcal{T}(x, y)), z)$.

4. $x \leq_{L^{*}} x^{\prime}, y \leq L^{*} y^{\prime} \Longrightarrow \mathcal{T}(x, y) \leq \operatorname{mathcalT}\left(x^{\prime}, y^{\prime}\right)$, (monotonicity).

Definition 2.4. [8] An decreasing mapping $\mathcal{N}: L^{*} \rightarrow L^{*}$ is called a negator on $L^{*}$ if it satisfying: $\mathcal{N}\left(0_{L^{*}}=1_{L^{*}}\right.$ and $\mathcal{N}\left(1_{L^{*}}=0_{L^{*}}\right.$. Further, $\mathcal{N}$ is called an involutive negator if for all $x \in L^{*}$ we have $\mathcal{N}(\mathcal{N}(x))=x$.

Aa decreasing mapping $\mathcal{N}:[0,1] \rightarrow[0,1]$ is called a negator on $[0,1]$ if it satisfying $\mathcal{N}(0)=1$ and $\mathcal{N}(1)=0$.

We denote by $\mathcal{N}_{s}$ for the standard negator on $[0,1]$ which is defined for all $x \in[0,1]$ by: $\mathcal{N}_{s}(x)=1-x$.

Definition 2.5. [24] Let $M, N: X^{2} \times(0, \infty) \rightarrow[0,1]$ be two fuzzy sets satisfying $M(x, y, t)+N(x, y, t) \leq 1$, for all $x, y \in X$ and $t>0$. The triplet $\left(X, \mathcal{M}_{M, N}, \mathcal{T}\right)$ is called to be an intuitionistic fuzzy metric space if $X$ is an arbitrary and non-empty set, $\mathcal{T}$ is a continuous $t$-representable and $\mathcal{M}_{M, N}$ is a mapping (an intuitionistic fuzzy set)from $X^{2} \times(0, \infty)$ into $L^{*}$ satisfying the following conditions for every $x, y \in X$ and $t, s>0$ :

(i) $\mathcal{M}_{M, N}\left((x, y,):.(0,1) \rightarrow L^{*}\right.$ is continuous,

(ii) $\mathcal{M}_{M, N}(x, y, t)=1_{L^{*}}$ if and only if $x=y$,

(iii) $\mathcal{M}_{M, N}(x, y, t)>_{L^{*}} 0_{L^{*}}$,

(iv) $\mathcal{M}_{M, N}(x, y, t)=\mathcal{M}_{M, N}(x, y, t)(y, x, t)$,

(v) $\mathcal{M}_{M, N}(x, y, t+s) \geq_{L^{*}} \mathcal{T}\left(\mathcal{M}_{M, N}(x, z, t)(x, z, t), \mathcal{M}_{M, N}(z, y, t)(z, y, s)\right)$.

Example 2.1. [24] Let $(X, d)$ be a metric space and $\left.\mathcal{T}(a, b)=\left(a_{1}, b_{1}\right), \min \left(a_{2}+b_{2}, 1\right)\right)$ for all $a=\left(a_{1}, a_{2}\right)$ and $b=$ $\left(b_{1}, b_{2}\right) \in L^{*} . M$ and $N$ are to fuzzy sets on $X^{2} \times(0, \infty)$ defined by:

$$
\mathcal{M}_{M, N}(x, y, t)=(M(x, y, t), N(x, y, t))=\left(\frac{h t^{n}}{h t^{n}+m d(x, y)}, \frac{m d(x, y)}{h t^{n}+m d(x, y)}\right),
$$

for all $t, h, m, n \in \mathbb{R}_{+} .\left(X, \mathcal{M}_{M, N}, \mathcal{T}\right)$ is modified intuitionistic fuzzy metric space. 


\section{Example 2.2. [24}

Let $X=\mathbb{N}$ and $\left.\mathcal{T}(a, b)=\left(\max \left(0, a_{1}+b_{1}-1\right), a_{2}+b_{2}-a_{2} b_{2}\right)\right)$ for all $a=\left(a_{1}, a_{2}\right)$ and $b=\left(b_{1}, b_{2}\right) \in L^{*}$. $M, N: X^{2} \times(0, \infty) \rightarrow L^{*}$ are two fuzzy sets such that:

$$
\mathcal{M}_{M, N}(x, y, t)=(M(x, y, t), N(x, y, t))= \begin{cases}\left(\frac{x}{y}, \frac{y-x}{y}\right), & \text { if } x \leq y \\ \left(\frac{y}{x}, \frac{x-y}{x}\right), & \text { if } y \leq x\end{cases}
$$

Then $\left(X, \mathcal{M}_{M, N}, \mathcal{T}\right)$ is modified intuitionistic fuzzy metric space.

Definition 2.6. [2] Let $(X, M, N, *, \diamond)$ be an intuitionistic fuzzy metric space and let $\left\{x_{n}\right\}$ be a sequence in $X$,

1. $\left\{x_{n}\right\}$ is convergent to some point $x$ in $X$, if $\lim _{n \rightarrow \infty} \mathcal{M}_{M, N}\left(x_{n}, x, t\right)=1_{L^{*}}$, for all $t>0$,

2. $\left\{x_{n}\right\}$ is a Cauchy sequence if, for each $0<\varepsilon<1$ and $t>0$, there exists $n_{0}$ such that

$$
\mathcal{M}_{M, N}\left(x_{n}, y_{m}, t\right)>\left(N_{\varepsilon}, \varepsilon\right),
$$

and for each $n, m \geq n_{0}$, where $N_{s}$ is the standard negator.

3. $(X, M, N, *, \diamond)$ is complete if every Cauchy sequence in it is convergent.

Lemma 2.2. [23] Let $\mathcal{M}_{M, N}$ be an intuitionistic fuzzy metric.Then, for any $t>0, \mathcal{M}_{M, N}(x, y, t)$ is non-decreasing with respect to $t$ in $\left(L^{*}, \leq_{L^{*}}\right)$, for all $x, y \in X$.

Definition 2.7. [24] Let $\left(X, \mathcal{M}_{M, N}, \mathcal{T}\right)$ be a modified IFMS. Then $\mathcal{M}_{M, N}$ is said to be continuous on $X \times X \times(0, \infty)$, if

$$
\lim _{n \rightarrow \infty} \mathcal{M}_{M, N}\left(x_{n}, y_{n}, t_{n}\right)=\mathcal{M}_{M, N}(x, y, t),
$$

whenever a sequence $\left(x_{n}, y_{n}, t_{n}\right)$ in $X \times X \times(0, \infty)$ converges to a point $(x, y, t) \in X \times X \times(0, \infty)$, that is $\lim _{n \rightarrow \infty} \mathcal{M}_{M, N}\left(x_{n}, x, t\right)=$ $\lim _{n \rightarrow \infty} \mathcal{M}_{M, N}\left(y_{n}, y, t\right)=1_{L^{*}}$, and $\lim _{n \rightarrow \infty} \mathcal{M}_{M, N}\left(x, y, t_{n}\right)=\mathcal{M}_{M, N}(x, y, t)$.

Lemma 2.3. [24] Let $\left(X, \mathcal{M}_{M, N}, \mathcal{T}\right)$ be a modified IFMS. Then $\mathcal{M}_{M, N}$ is a continuous function on $X \times X \times(0, \infty)$.

Definition 2.8. Mappings $A$ and $S$ on a modified IFMS $\left(X, \mathcal{M}_{M, N}, \mathcal{T}\right)$ are:

1. compatible [24] if

$$
\lim _{n \rightarrow \infty} \mathcal{M}_{M, N}\left(A S x_{n} n, S A x_{n}, t\right)=1_{L^{*}},
$$

for all $t>0$, whenever $\left\{x_{n}\right\}$ is a sequence in $X$ such that $\lim _{n \rightarrow \infty} A x_{n}=\lim _{n \rightarrow \infty} A x_{n}=z \in X$.

2. non compatible [29] if there exists at least one sequence $\left\{x_{n}\right\}$ in $X$ such that

$$
\lim _{n \rightarrow \infty} A x_{n}=\lim _{n \rightarrow \infty} S x_{n}=x \in X,
$$

but

$$
\lim _{n \rightarrow \infty} \mathcal{M}_{M, N}\left(A S x_{n}, S A x_{n}, t\right) \neq 1_{L^{*}},
$$

or non-existent for at least one $t>0$.

3. weakly compatible [24] if they commute at their coincidence points, i.e, if $A u=$ Su for some $u \in X$, then $A S u=$ SAu.

4. satisfy the property (E.A) [24] if there exists a sequence $\left\{x_{n}\right\}$ in $X$ such that for all $t>0$

$$
\lim _{n \rightarrow \infty} \mathcal{M}_{M, N}\left(A x_{n}, z, t\right)=\lim _{n \rightarrow \infty} \mathcal{M}_{M, N}\left(S x_{n}, z, t\right)=1_{L^{*}},
$$

for some $z \in X$.

Definition 2.9. [19] Two mappings $A$ and $S$ of a modified IFMS $\left(X, \mathcal{M}_{M, N} \mathcal{T}\right)$ are reciprocally continuous if

$$
\lim _{n \rightarrow \infty} \mathcal{M}_{M, N}\left(A S x_{n}, A z\right)=\lim _{n \rightarrow \infty} \mathcal{M}_{M, N}\left(S A x_{n}, S z, t\right)=1_{L^{*}}
$$

whenever $\left\{x_{n}\right\}$ is a sequence in $X$ such that

$$
\lim _{n \rightarrow \infty} A x_{n}=\lim _{n \rightarrow \infty} S x_{n}=z
$$

for some $z$ in $X$. 
Motivated by [3], we get:

Definition 2.10. Mappings ( $A$ and $S$ are weakly subsequentially continuous (wsc), if there exists a sequence $\left\{x_{n}\right\}$ satisfying

$$
\lim _{n \rightarrow \infty} A x_{n}=\lim _{n \rightarrow \infty} S x_{n}=z
$$

for some $z \in X$ and

$$
\lim _{n \rightarrow \infty} \mathcal{M}_{M, N}\left(A S x_{n}, A z, t\right)=1_{L^{*}}
$$

or

$$
\lim _{n \rightarrow \infty} \mathcal{M}_{M, N}\left(S A x_{n}, S z, t\right)=1_{L^{*}}
$$

In above definition if we have only

$$
\lim _{n \rightarrow \infty} \mathcal{M}_{M, N}\left(A S x_{n}, A z, t\right)=1_{L^{*}}
$$

then $(A, S)$ is called to be $A$-subsequentially continuous.

Example 2.3. Let $X=[0,2]$ and $\mathcal{M}_{M, N}(x, y, t)=\left(\frac{t}{t+|x-y|}, \frac{|x-y|}{t+|x-y|}\right)$ with $\mathcal{T}(a, b)=\left(a_{1} b_{1}, \min \left(a_{2}+b_{2}, 1\right)\right)$ for all $a=\left(a_{1}, a_{2}\right)$ and $b=\left(b_{1}, b_{2}\right) \in L^{*}$. Define $A, S$ as follows:

$$
A x=\left\{\begin{array}{ll}
1+x, & 0 \leq x \leq 1 \\
\frac{x+1}{2}, & 1<x \leq 2
\end{array} \quad, \quad S x= \begin{cases}1-x, & 0 \leq x \leq 1 \\
2-x, & 1<x \leq 2\end{cases}\right.
$$

It is clear the discontinuity of $A$ and $S$ at 1.

Let $\left\{x_{n}\right\}$ be a sequence in $X$ defined by: $x_{n}=\frac{1}{n}$, for each $n \geq 1$.

Clearly that

$$
\lim _{n \rightarrow \infty} \mathcal{M}_{M, N}\left(A x_{n}, 1, t\right)=\lim _{n \rightarrow \infty} \mathcal{M}_{M, N}\left(S x_{n}, 1, t\right)=1_{L^{a s t}}
$$

we have again:

$$
\lim _{n \rightarrow \infty} \mathcal{M}_{M, N}\left(A S x_{n}, A(1), t\right)=1_{L^{*}} .
$$

Hence $(A, S)$ is $A$-subsequentially continuous.

Motivated by [27, 28], define:

Definition 2.11. Self maps $A$ and $S$ of a modified $\operatorname{IFMS}\left(X, \mathcal{M}_{M, N}, \mathcal{T}\right)$ are said to be compatible of type (E), if

$$
\lim _{n \rightarrow \infty} \mathcal{M}_{M, N}\left(S^{2} x_{n}, A z, t\right)==\lim _{n \rightarrow \infty} \mathcal{M}_{M, N}\left(S A x_{n}, A z, t\right)=1_{L^{a s t}},
$$

and

$$
\lim _{n \rightarrow \infty} \mathcal{M}_{M, N}\left(A^{2} x_{n}, S z, t\right)=\lim _{n \rightarrow \infty} \mathcal{M}_{M, N}\left(A S x_{n}, S z, t\right)=1_{L^{*}},
$$

whenever $\left\{x_{n}\right\}$ is a sequence in $X$ satisfying:

$$
\lim _{n \rightarrow \infty} S x_{n}==\lim _{n \rightarrow \infty} A x_{n}=z,
$$

for some $z \in X$.

Definition 2.12. Two self mappings $A$ and $S$ of a modified IFMS $(X, \mathcal{M}, * T)$ into itself are said to be A-compatible of type (E), if

$$
\lim _{n \rightarrow \infty} \mathcal{M}_{M, N}\left(A^{2} x_{n}, S z, t\right)=\lim _{n \rightarrow \infty} \mathcal{M}_{M, N}\left(A S x_{n}, S z, t\right)=1_{L^{*}}
$$

Remark that if the pair $(A, S)$ is compatible of type (E), so it is $S$-compatible and $A$-compatible of type (E), but the converse may be not true.

Example 2.4. Let $X=\mathbb{R}_{+}$and $\mathcal{M}_{M, N}(x, y, t)=\left(\frac{t}{t+|x-y|}, \frac{|x-y|}{t+|x-y|}\right)$ with $\mathcal{T}(a, b)=\left(a_{1} b_{1}, \min \left(a_{2}+b_{2}, 1\right)\right)$ for all $a=\left(a_{1}, a_{2}\right)$ and $b=\left(b_{1}, b_{2}\right) \in L^{*}$. We define $A, S$ as follows:

$$
A x=\left\{\begin{array}{ll}
2, & 0 \leq x \leq 2 \\
x+1, & x>2
\end{array} \quad S x= \begin{cases}\frac{x+2}{2}, & 0 \leq x \leq 2 \\
0, & x>2\end{cases}\right.
$$


Consider the sequence $\left\{x_{n}\right\}$ which defined by: $x_{n}=2-\frac{1}{n}$, for all $n \geq 1$.

$$
\begin{gathered}
\lim _{n \rightarrow \infty} \mathcal{M}_{M, N}\left(S x_{n}, 2, t\right)=1_{L^{*}} \\
\lim _{n \rightarrow \infty} \mathcal{M}_{M, N}\left(A^{2} x_{n}, S(2), t\right)=\lim _{n \rightarrow \infty} \mathcal{M}_{M, N}(A S x n, S(2), t)=1_{L^{*}} \\
\lim _{n \rightarrow \infty} \mathcal{M}_{M, N}\left(S^{2} x_{n}, A(2), t\right)=\lim _{n \rightarrow \infty} \mathcal{M}_{M, N}(S A x n, A(2), t)=1_{L^{*}},
\end{gathered}
$$

then $(A, S)$ is compatible of type $(E)$.

Let $\Psi$ be the set of all continuous functions $F:\left(L^{*}\right)^{6} \rightarrow L^{*}$ satisfying the conditions:

$\left(F_{1}\right):$ For all $u, v \geq_{L^{*}} 0_{L^{*}}, F(u, v, u, v, v, u) \geq_{L^{*}} 0_{L^{*}}$, or $F(u, v, v, u, u, v) \geq_{L^{*}} 0_{L^{*}}$ implies that $u \geq_{L^{*}} v$.

$\left(F_{2}\right)$ : For all $u, v \geq_{L^{*}} 0_{L^{*}}, F(u, u, 1,1, u, u) \geq_{L^{*}} 0_{L^{*}}$ implies that $u \geq_{L^{*}} 1$,

where $u=\left(u_{1}, u_{2}\right), v=\left(v_{1}, v_{2}\right)$ and $1=1_{L^{*}}=(1,0)$.

Example 2.5.

$$
F\left(t_{1}, t_{2}, t_{3}, t_{4}, t_{5}, t_{6}\right)=15 t_{1}-13 t_{2}+5 t_{3}-7 t_{4}+t_{5}-t_{6}
$$

Example 2.6.

$$
F\left(t_{1}, t_{2}, t_{3}, t_{4}, t_{5}, t_{6}\right)=t_{1}-\frac{1}{2} t_{2}-\frac{5}{6} t_{3}+\frac{1}{3} t_{4}+t_{5}-t_{6}
$$

\section{Main results}

Theorem 3.1. Let $\left(X, \mathcal{M}_{M, N}, \mathcal{T}\right)$ be a modified intuitionistic fuzzy metric space, $A, B, S$ are self mappings on $X$, if

1. the pair $(A, S)$ is compatible of type $(E)$ and weakly subsequentially continuous and,

2. the pair $(B, T)$ is compatible of type $(E)$ and weakly subsequentially continuous.

Hence $(A, S)$ as well as $(B, T)$ have a coincidence point.

Moreover, the mappings $A, B, S$ and $T$ have a unique common fixed point provided for all $x, y \in X$ and $t>0$ we have:

$$
\begin{array}{r}
F\left(\mathcal{M}_{M, N}(A x, B y, t), \mathcal{M}_{M, N}(S x, T y, t), \mathcal{M}_{M, N}(A x, S x, t),\right. \\
\left.\mathcal{M}_{M, N}(B y, T y, t), \mathcal{M}_{M, N}(S x, B y, t), \mathcal{M}_{M, N}(T y, A x, t)\right) \geq_{L^{*}} 0_{L^{*}},
\end{array}
$$

Proof. Since the pair $(A, S)$ is wsc, there exists a sequence $\left\{x_{n}\right\}$ in $X$ such that $\lim _{n \rightarrow \infty} A x_{n}=\lim _{n \rightarrow \infty} S x_{n}=z$ for some $z \in X$ and $\lim _{n \rightarrow \infty} A S x_{n}=A z, \lim _{n \rightarrow \infty} S A x_{n}=S z$. Again $(A, S)$ is compatible of type $(\mathrm{E})$ implies that

$$
\lim _{n \rightarrow \infty} A S x_{n}=\lim _{n \rightarrow \infty} A^{2} x_{n}=S z
$$

and

$$
\lim _{n \rightarrow \infty} S A x_{n}=\lim _{n \rightarrow \infty} S^{2} x_{n}=A z
$$

consequently we obtain $A z=S z$ and $z$ is a coincidence point for $A$ and $S$. Similarly for $B$ and $T$, since $(B, T)$ is wsc (suppose that it is $B$-subsequentially continuous) there exists a sequence $\{y n\}$ such

$$
\lim _{n \rightarrow \infty} B y_{n}=\lim _{n \rightarrow \infty} T y_{n}=w
$$

for some $w \in X$ and

$$
\lim _{n \rightarrow \infty} B T y_{n}=B w .
$$

The pair $(B, T)$ is compatible of type (E) implies that

$$
\lim _{n \rightarrow \infty} B T y_{n}=\lim _{n \rightarrow \infty} B^{2} y_{n}=T w
$$




$$
\lim _{n \rightarrow \infty} T B y_{n}=\lim _{n \rightarrow \infty} T^{2} y_{n}=B w
$$

so we have $B w=T w$.

We claim $A z=B w$, if not by using (1)) we get:

$$
F\left(\mathcal{M}_{M, N}(A z, B w, t), \mathcal{M}_{M, N}(A z, B w, t), 1,1, \mathcal{M}_{M, N}(A z, B w, t), \mathcal{M}_{M, N}(A z, B w, t)\right) \geq_{L^{*}} 0_{L^{*}}
$$

and so from $\left(F_{2}\right)$, we get $\mathcal{M}_{M, N}(A z, B w, t)=1$. Hence $A z=B w$.

Now we will prove $z=A z$, if not by using (1)) we get:

$$
\begin{gathered}
F\left(\mathcal{M}_{M, N}\left(A x_{n}, B w, t\right) \mathcal{M}\left(S x_{n}, T w, t\right), 1\right. \\
\left.\left.\mathcal{M}_{M, N}\left(A x_{n}, S x_{n}, t\right), \mathcal{M}_{M, N}\left(S x_{n}, B w, t\right), \mathcal{M}_{M, N}\left(A x_{n}, T w, t\right)\right)\right) \geq_{L^{*}} 0_{L^{*}}
\end{gathered}
$$

letting $n \rightarrow \infty$ we get

$$
\begin{gathered}
F\left(\mathcal{M}_{M, N}(z, B w, t) \mathcal{M}(z, T w, t), 1,1, \mathcal{M}_{M, N}(z, B w, t), \mathcal{M}_{M, N}(z, T w, t)\right. \\
=F\left(\mathcal{M}_{M, N}(z, A z, t), \mathcal{M}(z, A z, t), 1,1, \mathcal{M}_{M, N}(z, A z, t), \mathcal{M}_{M, N}(z, A z, t) \geq_{L^{*}} 0_{L^{*}}\right.
\end{gathered}
$$

which implies that $\mathcal{M}_{M, N}(z, A z, t)=1_{L^{*}}$. Hence $z=A z$.

Now, We will show that $z=w$, if not by using (1), we get:

$$
\begin{gathered}
F\left(\mathcal{M}_{M, N}\left(A x_{n}, B y_{n}, t\right) \mathcal{M}\left(S x_{n}, T y_{n}, t\right), \mathcal{M}_{M, N}\left(A x_{n}, S_{x} n, t\right),\right. \\
\left.\mathcal{M}_{M, N}\left(B y_{n}, T y_{n}, t\right), \mathcal{M}_{M, N}\left(S x_{n}, B y_{n}, t\right), \mathcal{M}_{M, N}\left(A x_{n}, T y_{n}, t\right)\right) \geq_{L^{*}} 0_{L^{*}},
\end{gathered}
$$

letting $n \rightarrow \infty$ we get

$$
F\left(\mathcal{M}_{M, N}(z, w, t) \mathcal{M}(z, w, t), 1,1, \mathcal{M}_{M, N}(z, w, t), \mathcal{M}_{M, N}(z, w, t) \geq_{L^{*}} 0_{L^{*}}\right.
$$

then $\mathcal{M}_{M, N}(z, w, t)=1_{L^{*}}$. Hence $z=w$.

For the uniqueness, suppose $q$ another fixed point, by using (1) we get:

$$
F\left(\mathcal{M}_{M, N}(z, q, t) \mathcal{M}(z, q, t), 1,1, \mathcal{M}_{M, N}(z, q, t), \mathcal{M}_{M, N}(z, q, t) g e q_{L^{*}} 0_{L^{*}}\right.
$$

then $\mathcal{M}_{M, N}(z, q, t)=1$. Hence $z$ is unique.

If $A=B$ and $S=T$ we get the following corollary:

Corollary 3.1. Let $\left(X, \mathcal{M}_{M, N}, \mathcal{T}\right)$ be a modified IFMS and let $A, S$ two self mappings on $X$ into itself such the pair $(A, S)$ is wsc and compatible of type (E), then $A$ and $S$ have a coincidence point.

$A$ and $S$ have a unique common fixed point provided, for all $x, y \in X$ we have:

$$
\begin{gathered}
F\left(\mathcal{M}_{M, N}(A x, A y, t), \mathcal{M}_{M, N}(S x, S y, t), \mathcal{M}_{M, N}(A x, S x, t),\right. \\
\left.\mathcal{M}_{M, N}(A y, S y, t), \mathcal{M}_{M, N}(S x, A y, t), \mathcal{M}_{M, N}(A x, S y, t)\right) \geq_{L^{*}} 0_{L^{*}} .
\end{gathered}
$$

Corollary 3.2. The results of Theorem 3.1 remain true if the conditions (1) and (2) are replaced by the following:

$\left(1^{\prime}\right)$ the pair $(A, S)$ is A-subsequentially continuous and A-compatible of type (E) (or S-subsequentially continuous and $S$-compatible of type $(E))$,

$\left(2^{\prime}\right)$ the pair $(B, T)$ is B-subsequentially continuous and B-compatible of type (E) (or T-subsequentially continuous and $T$-compatible of type $(E)$ ).

Theorem 3.2. The results of Theorem 3.1 remain true if the conditions (1) and (2) are replaced by the following:

$\left(1^{\prime \prime}\right)$ the pair $(A, S)$ is subsequentially continuous and A-compatible(or $S$-compatible) of type $(E)$,

$\left(2^{\prime \prime}\right)$ the pair $(B, T)$ is subsequentially continuous and B-compatible(or T-compatible) of type (E).

Theorem 3.3. Let $\left(X, \mathcal{M}_{M, N}, \mathcal{T}\right)$ be a modified IMS and let $A, B, S$ be self mappings on $X$ satisfying (1), suppose

1. the pair $(A, S)($ or $(B, T))$ is wsc and compatible of type $(E)$, 
2. $\overline{A(X)} \subset T(X)($ or $\overline{B(X)} \subset S(X))$,

3. $(B, T)$ is weakly compatible (or $(A, S)$ is weakly compatible).

Then $A, B, S$ and $T$ have unique common fixed point in $X$.

Proof. Since the pair $(A, S)$ is wsc and compatible of type (E), there exists a sequence $\left\{x_{n}\right\}$ in $X$ such $\lim _{n \rightarrow \infty} A x_{n}=$ $\lim _{n \rightarrow \infty} S x_{n}=z$ for some $z \in X$ and $A z=S z$.

Moreover the subsequence $\left\{A x_{n}\right\}$ converges to $z$ in $\overline{A X}$ and since $\overline{A(X)} \subset T(X)$, so there exists $v \in X$ such $z=T v$, we show $z=B v$, if not by using (1) we get:

$$
\begin{aligned}
& F\left(\mathcal{M}_{M, N}\left(A x_{n}, B v, t\right), \mathcal{M}_{M, N}\left(S x_{n}, T v, t\right), \mathcal{M}_{M, N}\left(A x_{n}, S x_{n}, t\right),\right. \\
& \left.\mathcal{M}(B v, T v, t), \mathcal{M}_{M, N}\left(S x_{n}, B v, t\right), \mathcal{M}_{M, N}\left(A x_{n}, T v, t\right)\right) \geq_{L^{*}} 0_{L^{*}},
\end{aligned}
$$

letting $n \rightarrow \infty$, we get:

$$
F\left(\mathcal{M}_{M, N}(z, B v, t), 1,1, \mathcal{M}(B v, z, t), \mathcal{M}_{M, N}(z, B v, t), 1\right) \geq_{L^{*}} 0_{L^{*}}
$$

using $\left(F_{2}\right)$, we get

$$
\mathcal{M}_{M, N}(T v, B v, t)=1,
$$

then $T v=B v$ and so $v$ is a coincidence point for $B$ and $T$, the pair $(B, T)$ is weakly compatible implies that $B z=T z$.

We show that $A z=B z$, if not by using (1) we get

$$
F\left(\mathcal{M}_{M, N}(A z, B z, t), \mathcal{M}(S z, T z, t), 1,1, \mathcal{M}_{M, N}(S z, B z, t), \mathcal{M}_{M, N}(A z, T z, t)\right) \geq_{L^{*}} 0_{L^{*}}
$$

from $\left(F_{2}\right)$, we get

$$
\mathcal{M}_{M, N}(A z, B z, t) \geq_{L^{*}} 1 L^{*}
$$

for all $t>0$, which implies $\mathcal{M}_{M, N}(A z, B z, t)=1$ and so $A z=B z$.

Now, we claim $z=A z$, if not by using (1), we get:

$$
\begin{aligned}
& F\left(\mathcal{M}_{M, N}(A z, B v, t), \mathcal{M}(S z, T v, t), 1,1, \mathcal{M}_{M, N}(S z, B v, t), \mathcal{M}_{M, N}(A z, T v, t)\right)= \\
& F\left(\mathcal{M}_{M, N}(A z, z, t), \mathcal{M}(A z, z, t), 1,1, \mathcal{M}_{M, N}(A z, z, t), \mathcal{M}_{M, N}(A z, z, t)\right) \geq_{L^{*}} 0_{L^{*}},
\end{aligned}
$$

using $\left(F_{2}\right)$, we get

$$
\mathcal{M}_{M, N}(A z, z, t) \geq_{L^{*}} 1 L^{*}
$$

for all $t>0$, then $\mathcal{M}_{M, N}(A z, z, t)=1$ and $z$ is a common fixed point for $A, B, S$ and $T$.

For the uniqueness, it is similar as in proof of Theorem 3.1.

Theorem 3.4. Let $\left(X, \mathcal{M}_{M, N}, \mathcal{T}\right)$ be a modified IFMS and let $A, B, S$ be self mappings a on $X$ satisfying (1), suppose

1. the pair $(A, S)($ or $(B, T))$ is wsc and compatible of type $(E)$,

2. $A(X) \subset T(X)($ or $B(X) \subset S(X))$,

3. $\left\{B y_{n}\right\}$ converges for every sequence $\left\{y_{n}\right\}$ in $X$, whenever $\left\{T y_{n}\right\}$ converges( or $\left\{A x_{n}\right\}$ converges for every sequence $\left\{x_{n}\right\}$ in $X$, whenever $\left\{S x_{n}\right\}$ converges,

4. $(B, T)$ is weakly compatible (or $(A, S)$ is weakly compatible).

Then $A, B, S$ and $T$ have unique common fixed point in $X$.

Proof. As in proof of Theorem 3.1 if the pair $(A, S)$ is wsc and compatible of type (E), then there exists a sequence $\left\{x_{n}\right\}$ in $X$ satisfying $\lim _{n \rightarrow \infty} A x_{n}=\lim _{n \rightarrow \infty} S x_{n}=z$ for some $z \in X$ and $A z=S z$.

The inclusion $A(X) \subset T(X)$ implies that there exists a sequence $\left\{y_{n}\right\}$ in $X$ such $\lim _{n \rightarrow \infty} A x_{n}=\lim _{n \rightarrow \infty} T y_{n}=z$.

We will show $\lim _{n \rightarrow \infty} B y_{n}=z$, if not by using (1) we get:

$$
\begin{gathered}
F\left(\mathcal{M}_{M, N}\left(A x_{n}, B y_{n}, t\right), \mathcal{M}\left(S x_{n}, T y_{n}, t\right) \mathcal{M}_{M, N}\left(A x_{n}, S x_{n}, t\right)\right. \\
\left.\mathcal{M}_{M, N}\left(B y_{n}, T y_{n}, t\right), \mathcal{M}_{M, N}\left(S x_{n}, B y_{n}, t\right), \mathcal{M}_{M, N}\left(A x_{n}, T y_{n}, t\right)\right) \geq_{L^{*}} 0_{L^{*}}
\end{gathered}
$$


letting $n \rightarrow \infty$ we get

$$
F\left(\mathcal{M}_{M, N}\left(z, \lim _{n \rightarrow \infty} B y_{n}, t\right), 1,1, \mathcal{M}_{M, N}\left(\lim _{n \rightarrow \infty} B y_{n}, z, t\right), \mathcal{M}_{M, N}\left(\lim _{n \rightarrow \infty} B y_{n}, z, t\right), 1\right) \geq_{L^{*}} 0_{L^{*}}
$$

from $\left(F_{1}\right)$, we obtain $\mathcal{M}_{M, N}\left(z, \lim _{n \rightarrow \infty} B y_{n}, t\right)=1$. Hence $\lim _{n \rightarrow \infty} B y_{n}=z$.

Now, we will prove $z=A z=S z$, if not by using 1 we get:

$$
\begin{gathered}
F\left(\mathcal{M}_{M, N}\left(A z, B y_{n}, t\right), \mathcal{M}\left(S z, T y_{n}, t\right), 1, \mathcal{M}\left(B y_{n}, T y_{n}, t\right),\right. \\
\left.\mathcal{M}_{M, N}\left(S z, B y_{n}, t\right), \mathcal{M}_{M, N}\left(A z, T y_{n}, t\right)\right) \geq_{L^{*}} 0_{L^{*}}
\end{gathered}
$$

letting $n \rightarrow \infty$ we get:

$$
F\left(\mathcal{M}_{M, N}(A z, z, t), \mathcal{M}(A z, z, t), 1,1, \mathcal{M}_{M, N}(A z, z, t), \mathcal{M}_{M, N}(A z, z, t)\right) \geq_{L^{*}} 0_{L^{*}}
$$

from $\left(F_{2}\right)$, we get $\mathcal{M}_{M, N}(A z, z, t) \geq_{L^{*}}=1_{L^{*}}$, for all $t>0$. Hence $z$ is a common fixed point for $A$ and $S$.

Since $A(X) \subset T(X)$, there exists $w \in X$ such $z=A z=T w$, we will sow $T w=B w$, by putting $x=z$ and $y=w$ in(1), we get:

$$
\begin{gathered}
F\left(\mathcal{M}_{M, N}(T w, B w, t), 1,1, \mathcal{M}(B w, T w, t), \mathcal{M}_{M, N}(T w, B w, t), 1\right)= \\
F\left(\mathcal{M}_{M, N}(A z, B w, t), 1,1, \mathcal{M}(B w, T w, t), \mathcal{M}_{M, N}(S z, B w, t), \mathcal{M}_{M, N}(T w, A z, t)\right) \geq_{L^{*}} 0_{L^{*}},
\end{gathered}
$$

using $\left(F_{2}\right)$, we get $\mathcal{M}_{M, N}(T w, B w, t) \geq_{L^{*}} 1_{L^{*}}$ for all $t>0$, then:

$$
\mathcal{M}_{M, N}(T w, B w, t)=1_{L^{*}},
$$

which implies that $T w=B w$.

The pair $(B, T)$ is weakly compatible, so $z=B z=T z$ and consequently $z$ is a common fixed point for $A, B, S$ and $T$.

For the uniqueness, it is similar as in proof of Theorem 3.1 .

Example 3.1. Let $\left(X, \mathcal{M}_{M, N}, \mathcal{T}\right)$ be a modified intuitionistic fuzzy metricspace, with $X=[0,1]$.For all $a=\left(a_{1}, a_{2}\right)$ and $b=\left(b_{1}, b_{2}\right) \in L^{*}$, and each $t \in(01)$ let $\left.\mathcal{T}(a, b)=\left(a_{1} b_{1}\right), \min \left(a_{2}+b_{2}, 1\right)\right)$, define:

$$
\mathcal{M}(A z, z, t)=\left(\frac{t}{t+|x-y|}, \frac{|x-y|}{t+|x-y|}\right)
$$

. Define self mappings $A, B, S$ and $T$ by: $A x=B x=1$,

$$
S x=T x= \begin{cases}1, & x \text { is rational } \\ \frac{1}{4}, & x \text { is irrational }\end{cases}
$$

Consider a sequence $\left\{x_{n}\right\}$ such for each $n \geq 1$ we have:

$x_{n}=\frac{1}{n}$, clearly that $\lim _{n \rightarrow \infty} A x_{n}=\lim _{n \rightarrow \infty} S x_{n}=1$, also we have:

$$
\begin{aligned}
& \lim _{n \rightarrow \infty} A S x_{n}=A(1)=1 \\
& \lim _{n \rightarrow \infty} A^{2} x_{n}=S(1)=1,
\end{aligned}
$$

then $(A, S)$ is A-subsequentially continuous and A-compatible of type $(E)$.

Taking $F\left(t_{1}, t_{2}, t_{3}, t_{4}, t_{5}, t_{6}\right)=t_{1}--\frac{1}{2} t_{2}-\frac{5}{6} t_{3}+\frac{1}{3} t_{4}+t_{5}-t_{6}$, then we will check that the inequality (1) is satisfied: for $x, y \in[0,1]$, we have:

$$
\begin{aligned}
& F\left(t_{1}, t_{2}, t_{3}, t_{4}, t_{5}, t_{6}\right)=(1,0)-\frac{1}{2}(1,0)+\frac{5}{6}(1,0)-\frac{1}{3}(1,0)-(1,0)+(1,0) \\
&=(0,0) \geq_{L^{*}} 0_{L^{*}} .
\end{aligned}
$$

Hence all hypotheses of Theorem 3.1 are satisfied, therefore 1 is the unique common fixed point for A and S. 


\section{REFERENCES}

[1] A. T. Atanassov, Intuitionistic fuzzy sets, Fuzzy Sets Syst. 20 (1986), 87-96.

[2] C.Alaca, D.Tukoglu and C.Yildiz, Fixed points in intuitionistic fuzzy metric spaces, Chaos, Solinons.Fractal. (2006),29pp.

[3] S.Beloul, Common fixed point theorems for weakly subsequentially continuous generalized contractions with aplications, Appl. Maths. E-Notes, 15 (2015), 173-186.

[4] H. Bouhadjera and C.G.Thobie,Common fixed point theorems for pairs of subcompatible maps,arXiv:0906.3159v1 [math.FA],(2009).

[5] S.Chauhan, M. Imdadb and B. Samet,Coincidence and common fixed point theorems in modified intuitionistic fuzzy metric spaces, Math. Comp Model 58 (2013),892-900.

[6] D. Çoker, An introduction to intuitionistic fuzzy topological spaces, Fuzzy Sets and Systems 88 (1997), 81-99.

[7] G.Deschrijver and E.E.Kerre, On the relationship between some extensions of fuzzy set theory, Fuzzy Sets Syst. 133, (2003), 227-235.

[8] G.Deschrijver, c.Cornelis and E.E.Kerre,On the representation of intuitionistic fuzzy $t$-norms and t-conorms, IEEE Trans Fuzzy Syst. 12, 45-61 (2004).

[9] A. George, P. Veeramani, On some results in fuzzy metric spaces, Fuzzy Sets Systems 64 (3) (1994) $395-399$.

[10] V. Gregori, S. Romaguera and P. Veereamani, A note on intuitionistic fuzzy metric spaces, Chaos, Solitons .Fractals. 28 (2006), 902-905.

[11] D.Gopal and M. Imdad,Some new common fixed point theorems in fuzzy metric spaces, Ann. Univ. Ferrara Sez. VII Sci. Mat. 57(2) (2011), 303-316.

[12] X. Huang, C. Zhu, Xi Wen, Common fixed point theorems for families of compatible mappings in intuitionistic fuzzy metric spaces, Ann. Univ. Ferrara Sez. VII Sci. Mat. 56 (2) (2010) 305-326.

[13] M.Imdad, J. Ali and M. Tanveer, Remarks on some recent metrical common fixed point theorems, Appl. Math. Lett. 24(2011), 1165-1169.

[14] M. Imdad, J. Ali and M. Hasan,Common fixed point theorems in modified intuitionistic fuzzy metric spaces,Ira.J. of Fuzzy Syst Vol. 9, No. 5, (2012) pp. 77-92.

[15] S. Jain and L.B. Jain, Compatibility of type $(P)$ in modified intuitionistic fuzzy metric space, J. Nonlinear Sci. Appl. 3 (2) (2010) $96 ? 09$.

[16] G. Jungck,Compatible mappings and common fixed points, Int. J. Math. Math. Sci. 9 (1986) 771-779.

[17] G. Jungck and B.E Rhoades, Fixed point for set valued functions without continuity, Indian J. Pure Appl. Math. 29 (3),(1998) 227-238.

[18] S. Kumar, Common fixed point theorems in intuitionistic fuzzy metric spaces using property (E.A), J. Indian Math. Soc. (N.S.) 76 (1?) (2009) 93-104.

[19] S.Manro, On Common Fixed Point Theorem for Weakly Compatible Maps Satisfying Common Property (E.A) and Implicit relation in Intuitionistic Fuzzy Metric Spaces,Int. J.Nonlinear Anal. Appl. 6 No 1 (2015), 1-8.

[20] U.Mishra,A.S. Ranadive and D.Gopal, Some fixed points theorems in fuzzy metric spaces,Tamkang J. Math. 39(4) (2008),309-316.

[21] J.H. Park, Intuitionistic fuzzy metric spaces, Chaos Solitons Fractals 22 (5) (2004) 1039-1046.

[22] I.Kramosil and J.Michalek,Fuzzy metric and statistical metric spaces,Kybernetica 11 (1975), 336-344.

[23] R. Saadati and J. H. Park, On the intuitionistic fuzzy topological spaces, Chaos,Solitons. Fractals 27 (2006), 331 ?44. 
[24] R.Saadati,S. Sedgi and N.Shobe, Modified intuitionistic fuzzy metric spaces and some fixed point theorems, Chaos Solitons and Fractals. 38, (2008), 36-47.

[25] B. Schweizer and S. Sklar,Statistical metric spaces, Pacific J. Math. 10 (1960) 313-334.

[26] S. Sedghi, N. Shobe, A. Aliouche, Common fixed point theorems in intuitionistic fuzzy metric spaces through conditions of integral type, Appl. Math.Inf. Sci. 2 (1) (2008) $61 ? 2$.

[27] M.R. Singh and Y. Mahendra Singh Compatible mappings of type (E) and common fixed point theorems of Meir-Keeler type, International J. Math. Sci. Engg. Appl. 1 (2),(2007) 299-315.

[28] M.R. Singh and Y. Mahendra Singh,On various types of compatible maps and common fixed point theorems for noncontinuous maps, Hacet. J. Math. Stat.40(4),(2011) 503 - 513.

[29] M. Tanveer, M. Imdad, D. Gopal, D.K. Patel, Common fixed point theorems in modified intuitionistic fuzzy metric spaces with common property (E.A.), Fixed Point Theory Appl. 2012 (2012) 36.

[30] L. A. Zadeh, Fuzzy sets, Inform. and control 89 (1965),3. 